\title{
Segurança da aplicabilidade da eletroestimulação neuromuscular na hemodinâmica de pacientes nas UTI's como prevenção da polineuromiopatia: uma revisão de
}

\section{literatura}

Safety of the applicability of neuromuscular electro-stimulation in hemodynamics of patients in

ICU's as a prevention of polyuromyopathy: a literature review

Seguridad de la aplicabilidad de la electrostimulación neuromuscular en hemodinámica de pacientes en UCI como prevención de poliuromiopatía: revisión de la literatura

Lisiane Lima Felix ORCID: https://orcid.org/0000-0002-3044-3465 Faculdade Facottur, Brasil E-mail: liz.limafelix@gmail.com.com Cyntia Fonseca do Rêgo Barros e Albuquerque ORCID: https://orcid.org/0000-0002-2576-5275 Faculdade Facottur, Brasil

E-mail: cyntiafrbalbuquerque15@gmail.com Harrison Euller Vasconcelos Queiroz ORCID: https://orcid.org/0000-0001-7906-2981 Faculdade Facottur, Brasil

E-mail: harrisoneuller@gmail.com

Flávia Carolina Lasalvia da Silva ORCID: https://orcid.org/0000-0003-3441-3201 Faculdade Facottur, Brasil E-mail: flaviaclasalvia@gmail.com

Eudson José Santos do Monte ORCID: https://orcid.org/0000-0002-1871-6619 Faculdade Facottur, Brasil E-mail: eudson41@gmail.com

Amanda Fonseca do Nascimento ORCID: https://orcid.org/0000-0001-7804-671X Faculdade Facottur, Brasil

E-mail: amandas2nascimento@gmail.com

Ingrid Larissa da Silva Laurindo ORCID: https://orcid.org/0000-0001-7658-2455 Faculdade Facottur, Brasil

E-mail: larissasilvalaurindo@gmail.com

Mateus Porfirio Rodrigues ORCID: https://orcid.org/0000-0002-6747-4898 Faculdade Facottur, Brasil

E-mail: mateusporfirio6@gmail.com

Camila Ellen Pinheiro dos Santos ORCID: https://orcid.org/0000-0002-8434-5342 Faculdade Facottur, Brasil

E-mail: camilaellen08@gmail.com

Manoela Gouveia dos Passos ORCID: https://orcid.org/0000-0002-0552-8408 Faculdade Facottur, Brasil

E-mail: manugouveia264@gmail.com

Klyvia Juliana Rocha de Moraes ORCID: https://orcid.org/0000-0001-7407-4027 Faculdade Facottur, Brasil

E-mail: klyviaj@yahoo.com.br

José Carlos Nogueira Nóbrega Júnior ORCID: https://orcid.org/0000-0003-3908-9260 Faculdade Facottur, Brasil E-mail: c10carlo@gmail.com 


\begin{abstract}
Resumo
Objetivo: Asseverar a segurança da aplicabilidade da eletroestimulação neuromuscular em pacientes nas UTI's como prevenção da polineuromiopatia. Métodos: O presente estudo expõe-se como uma revisão de literatura, com as buscas realizadas nas bases de dados PubMed, SciELO, Medline e Cochrane desde o início de março até abril de 2020, não havendo restrição de idiomas, sendo sua análise descritiva realizada no período de maio a junho do mesmo ano. Foram inseridos artigos que contivessem informações sobre segurança e efeitos da aplicabilidade da técnica nos desfechos, levando-se em conta as possíveis alterações de hemodinâmica. Resultados: Foram identificados 234 estudos relevantes, dos quais 215 foram excluídos por não apresentarem desfechos de interesse e segurança, e inclusos 5 ensaios clínicos randomizados. O tamanho da amostra variou entre 11 e 54 pacientes de ambos os sexos, com faixa etária variando entre 18 e 82 anos, fazendo uso ou não de ventilação mecânica invasiva, submetidos à estimulação elétrica neuromuscular em pacientes graves. Dos artigos selecionados, todos analisaram a influência da estimulação elétrica neuromuscular e não constataram efeitos adversos que influenciassem no estado clínico do paciente. Conclusão: Os resultados das análises sugerem que a eletroestimulação neuromuscular (EENM) pode ser implementada nas UTI's para recuperação e prevenção da polineuromiopatia, pois a ausência de efeitos secundários maléficos ou significativos enfatiza tal afirmação.
\end{abstract}

Palavras-chave: Fraqueza muscular; Unidade de Terapia Intensiva; Estimulação elétrica.

\begin{abstract}
Aim: To assert the safety of using neuromuscular electro-stimulation on ICU patients as a way to prevent polyneuromyopathy. Methods: This study comes as a literature revision with researches concentrated on databases such as PubMed, SciELO, Medline and Cochrane from early March until Abril 2020, with no idiom restrictions, and with its main analysis being written between the months of May and June. There have been inserted articles with informations on the safety and effects of the applicability of the technic in the outcomes, taking in consideration possible hemodynamics alterations. Results: There have been identified 234 relevant studies, from which 215 have been excluded for not presenting relevant and safe outcomes, and there were included 5 randomized clinical trials. Sample size has varied between 11 and 54 patients from both genders, in an age range from 18 to 82 years old, involving or not the use of invasive mechanical ventilation, who were submitted to neuromuscular electrical stimulation in critically ill patients. Amongst the selected articles, all of them analyzed the influence from the neuromuscular electrical stimulation and didn't recognize side effects that could influence in the patient's clinical condition. Conclusion: The analysis results suggest that the neuromuscular electro-stimulation (NMES) could be implemented in ICUs for the recovery and prevention of polyneuromyopathy, hence the lack of malefic or relevant side effects.
\end{abstract}

Keywords: Muscle weakness; Intensive Care Unit; Electrical stimulation.

\title{
Resumen
}

Objetivo: Afirmar la seguridad de la aplicabilidad de la estimulación eléctrica neuromuscular en pacientes de UCI como prevención de polineuromiopatía. Métodos: El presente estudio se presenta como una revisión de la literatura, con búsquedas realizadas en las bases de datos PubMed, SciELO, Medline y Cochrane desde principios de marzo a abril de 2020, no existe restricción de idioma, y su análisis descriptivo se realizó de mayo a junio del mismo año. Se insertaron artículos que contienen información sobre seguridad y los efectos de la aplicabilidad de la técnica en los resultados, teniendo en cuenta los posibles cambios hemodinámicos. Resultados: Se identificaron 234 estudios relevantes, de los cuales 215 fueron excluidos por no presentar resultados de interés y seguridad, e incluyó 5 ensayos clínicos aleatorizados. El tamaño de la muestra osciló entre 11 y 54 pacientes de ambos sexos, con edades comprendidas entre 18 y 82 años, utilizando o no ventilación mecánica invasiva, sometida a estimulación eléctrica neuromuscular en pacientes críticos. De los artículos seleccionados, todos analizaron la influencia de la estimulación eléctrica neuromuscular y no encontraron efectos adversos que influyeran en el estado clínico del paciente. Conclusión: Los resultados de los análisis sugieren que la electrostimulación neuromuscular (EENM) se puede implementar en las UCI para la recuperación y prevención de la polineuropatía, ya que la ausencia de efectos secundarios nocivos o significativos enfatiza esta afirmación.

Palabras clave: Debilidad muscular; Unidad de Terapia Intensiva; Estimulación eléctrica.

\section{Introdução}

Entende-se como fraqueza muscular adquirida em unidade de terapia intensiva, também denominada como polineuromiopatia, um enfraquecimento bilateral e simétrico que afeta os músculos periféricos e respiratórios, podendo já ser observado no segundo dia após a internação. É uma complicação significativa e comum em pacientes internados na unidade de terapia intensiva (UTI) comprometendo a qualidade de vida do paciente, mesmo após a alta hospitalar, ou levá-lo à óbito (Ferreira, et al., 2014; Godoy, et al., 2015).

Com o avanço da tecnologia e dos cuidados de terapia intensiva, foi permitido ao paciente crítico sobreviver e recuperar- 
se, porém, quando a internação é prolongada, poderá tornar-se prejudicial, havendo perda da massa muscular devido à restrição no leito. A imobilidade no leito tem um efeito direto e maléfico aos músculos periféricos e respiratórios, que contribui para o surgimento da polineuromiopatia em pacientes críticos, cujo fatores de risco estão associados à ventilação mecânica prolongada (Hashem, et al., 2016; França, et al., 2012; Puthucheary, et al., 2010).

Para o diagnóstico de fraqueza muscular periférica na UTI é mais comumente utilizado o escore de força muscular denominado Medical Research Council (MRC) ou de preensão palmar por meio da dinamometria, mas, para realização de ambos os escores, é preciso que o paciente seja colaborativo. Em pacientes incapazes de cooperar, o diagnóstico de polineuromiopatia fica limitado devido à dificuldade da disponibilidade da eletromiografia (EMG) e do estudo da condução nervosa (NCS) outros meios para avaliação da fraqueza muscular (Menezes, et al., 2018; Santos, et al., 2017; Puthucheary, et al., 2010).

A intervenção dos fisioterapeutas intensivistas busca alternativas na resolução dessa síndrome, utilizando alguns recursos como: a mobilização do paciente crítico e a eletroestimulação neuromuscular (EENM), com o objetivo de abrandar a incidência da polineuromiopatia com a redução no tempo de ventilação mecânica e facilitando o desmame ventilatório (Godoy, et al., 2015).

É essencial dar importância ao fato de que alguns pacientes da UTI acham-se entorpecidos, com alterações hemodinâmicas ou com diminuição da cognição sem a possibilidade de realizar movimentos ativos, portanto a EENM mostrase como um dos recursos alternativos para melhorar ou manter a força muscular, através de agentes eletrofísicos, cujo são agentes não invasivos que fazem uso de correntes elétricas, mudanças de temperatura e princípios de fotomodulação para alcançar efeitos terapêuticos (Sachetti, et al., 2018; Santos, et al., 2020).

A EENM é um mecanismo modulador que atua no fortalecimento e recuperação do músculo através da excitação do nervo periférico, permitindo que o músculo realize contrações involuntárias e eficientes através da corrente elétrica de baixa voltagem, aumentando o fluxo circulatório, a força e a resistência, além da redução da fadiga, dessa forma, prevenindo o decréscimo da massa muscular em pacientes sedados nas UTIs que se encontram-se em ventilação mecânica prolongada. Desse modo, a EENM torna-se uma alternativa possível para a prevenção da redução da força muscular e suas consequências, mantendo e/ou aumentando a de força e resistência (Meesen, et al., 2010; Santos, et al.,2014; Figueira, et al., 2021).

Por ser um mecanismo de recurso terapêutico alternativo e promissor na reabilitação, a EENM tem como principais vantagens a melhorara da funcionalidade motora com aumento ou manutenção da força muscular em pacientes críticos e prevenção da perda de sarcômeros ocasionada pelo desuso. Contudo, em contrapartida pode apresentar risco de queimaduras em casos de parâmetros errados, devendo ser aplicada apenas por profissionais treinados que utilizem parâmetros baseados em evidências (Sachetti et al.,2018; Santos et al.,2014).

Diante de uma comorbidade adquirida na UTI, como a fraqueza muscular, que é ocasionada pela atrofia da musculatura esquelética, devido ao imobilismo no leito, gerando assim uma redução da funcionalidade geral. Com isto, o objetivo do estudo versa asseverar a segurança da aplicabilidade da eletroestimulação neuromuscular na hemodinâmica de pacientes nas UTI's como prevenção da polineuromiopatia.

\section{Metodologia}

Trata-se de uma revisão de literatura realizada nas bases de dados PubMed, SciELO, Medline e Cochrane. Foram incluídos artigos que contivessem informações sobre segurança da aplicabilidade da técnica como desfecho, levando-se em conta as possíveis alterações de hemodinâmica e efeitos adversos.

Foram incluídos ensaios clínicos randomizados (ECR) com pacientes internados em UTI's, que estavam fazendo uso ou não de ventilação mecânica invasiva (VMI), conscientes ou não, todos submetidos à aplicação da eletroestimulação neuromuscular em grupos musculares periféricos e respiratórios acessórios, àqueles artigos que incluíram a descrição da 
intervenção clínica e que compararam os protocolos de sua aplicabilidade a fim de determinar sua eficiência, os que apresentaram desfechos relativos a sua eficácia e segurança da aplicabilidade da EENM e artigos publicados a partir do ano de 2015 à maio de 2020. Fizeram parte dos critérios de exclusão aqueles que envolveram estudos com crianças ou animais e relatos de caso.

O processo de busca e análise dos artigos foi realizado durante o período de março a maio de 2020, utilizando os seguintes descritores combinados nas línguas portuguesa e inglesa: "fraqueza muscular" (muscle weakness); "polineuromiopatia por doença crítica" (critical illness polyneuromyopathy), "unidade de terapia intensiva" (intensive care unit); "estimulação elétrica" (electrical stimulation); "efeitos adversos" (adverse effects) utilizando os descritores booleanos AND e OR. As buscas foram realizadas por duas pesquisadoras que, de forma independente, selecionaram os artigos a partir dos títulos, ano de publicação e leitura dos resumos, e os que, em seu resumo, não forneceram informações suficientes foram excluídos.

Posteriormente, os artigos selecionados foram confrontados, havendo ainda uma nova seleção para definição dos que iriam ser lidos na íntegra. Para sua análise, foi determinado um roteiro para escolha, sendo selecionados os que fossem ensaios clínicos randomizados com pacientes internados na UTI que estavam fazendo uso ou não da VMI, com protocolos de intervenção definidos, havendo comparação, efeitos encontrados e desfechos. O desfecho foram os efeitos acarretados pela aplicabilidade da EENM quanto a hemodinâmica, funcionalidade e segurança. A análise dos estudos selecionados e sua síntese foram realizadas de forma descritiva. Por fim, interpretados os resultados e efeitos sem manipulação ou intervenção das pesquisadoras com o intuito de delinear o conhecimento produzido.

\section{Resultados}

Foram identificados 234 artigos dos quais, após a leitura dos títulos e resumos, foram pré-selecionados 19 artigos para leitura completa. Os que foram excluídos justifica-se por não apresentarem os desfechos de interesse, segurança, ou não serem ensaios clínicos randomizados, resultando em um total de 5 artigos para análise e descrição detalhada por parte dos revisores e de forma independente (Figura 1).

O tamanho da amostra dos cinco estudos variou de 11 a 54 pacientes, totalizando 151 participantes de ambos os sexos com idades superiores a 18 anos até aos 82 anos. Todos em estado crítico e internados na unidade de terapia intensiva e que estavam fazendo uso ou não de VMI, estando conscientes ou não. Sendo divididos entre grupos controle e intervenção. Com relação a patologia ou motivo de internação: Akar, et al. (2015) se referiram a pacientes ( $\mathrm{n}=30$ ) com DPOC; o estudo de Stefanou, et al. (2016) referem-se a pacientes ( $\mathrm{n}=32)$ enfermos apresentando sepse não definindo qual patologia primária; Fischer, et al., (2016) citaram que eram pacientes críticos pós-cirúrgia cardiotorácica ( $\mathrm{n}=54)$; já o estudo de Silva, et al. (2017) que eram compostos por $82 \%$ de pacientes ( $\mathrm{n}=9$ ) com Politrauma, exceto com fraturas em MMII, por 9\% com Pancreatite ( $\mathrm{n}=1)$ e $1 \%$ por cirurgia abdominal $(\mathrm{n}=1)$ e por fim o artigo de Sachetti, et al. (2018) citaram apenas que eram pacientes críticos nas UTI's fazendo uso VMI ( $\mathrm{n}=24)$.

Todos os estudos envolvidos nesta revisão, realizaram programas de intervenções com grupos que pudessem fazer comparações. Entre os ensaios clínicos randomizados aqui analisados, os artigos de Fisher, et al. (2016) e o de Sachetti, et al. (2018) compararam o uso da EENM entre os grupos de controle e de intervenção; o estudo de Stefanou, et al. (2016) compararam grupos com uso da EENM com frequências diferentes; Akar, et al. (2015) separam os pacientes randomizados em três grupos nos quais o grupo 1 combinou exercícios ativos com EENM, o grupo 2 apenas EENM e grupo 3, exclusivamente, exercícios ativos. E, por fim, Silva, et al. (2017) avaliaram, como desfecho primário a segurança de um protocolo da EENM aplicados em cinco grupos musculares em pacientes críticos submetidos à VM. 
Figura 1 - Fluxograma mostrando as estratégias de buscas dos dados para revisão da literatura.

\section{Critérios de Revisão}

- Descritores: Fraqueza muscular; Unidade de terapia intensiva; Estimulação elétrica; Efeitos adversos.

- Base de dados: PubMed; Scielo; Medline e Cochrane.

- Inclusão: ECR; Internados UTI; Aplicação de EENM

* Periodo de busca e análise dos artigos: março a junho de 2020.

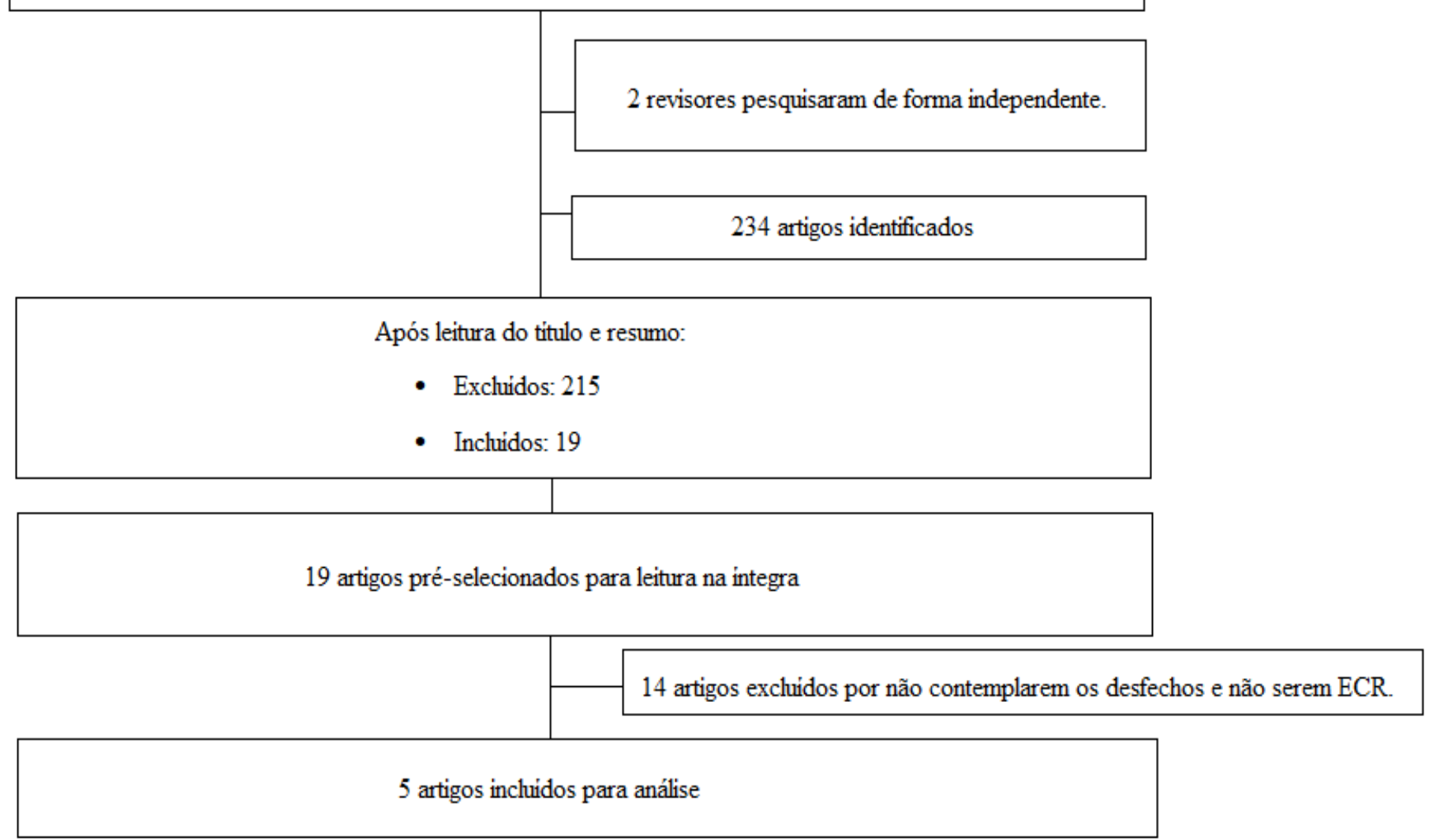

Fonte: Autores (2021).

Quanto ao programa de intervenção da EENM, este foi realizado no ambiente da UTI como prevenção da polineuromiopatia e segurança da sua aplicabilidade. O tempo de aplicabilidade da EENM variou apenas em 1 artigo, Silva, et al. (2017) com aplicação de 3 ciclos de 15 minutos totalizando 45 minutos na sessão e os demais estudos por 30 minutos a sessão; o número de sessões variou de 1 à 2 vezes ao dia e suas frequências variaram de $45 \mathrm{~Hz}, 50 \mathrm{~Hz}, 66 \mathrm{~Hz}, 75 \mathrm{~Hz}$ e $100 \mathrm{~Hz}$. A frequência entre os artigos variou entre a sua aplicabilidade, sendo que Akar, et al. (2015) e os estudos de Sachetti, et al. (2018) apresentaram a mesma frequência de 50Hz. O único artigo que utilizou frequências diferentes entre os grupos foi o de Stefanou, et al. (2016) aplicando respectivamente $45 \mathrm{~Hz}$ e $75 \mathrm{~Hz}$. A largura de pulso não foi referida em todos os artigos. Os que a mencionaram variou entre $300 \mu \mathrm{s}$ e $400 \mu \mathrm{s}$, sendo fixa durante toda as sessões. Silva, et al. (2017) aplicaram, em seu estudo, inicialmente, uma largura de pulso de $100 \mu \mathrm{s}$, sendo aumentada de $20 \mu$ s até $1000 \mu$ s a cada 1s até que houvesse uma contração visível. 
Dos cinco estudos incluídos, todos analisaram a influência da EENM na hemodinâmica como desfecho primário para determinar o quesito segurança, sendo que Silva, et al. (2017) avaliaram o quesito segurança, dentre outros níveis séricos, as aferições dos níveis de fosfoquinase (CPK) para avaliar o grau de lesão muscular. Fisher, et al. (2016) avaliaram a aplicação da EENM como requisito de recuperação da força muscular e impedimento da perda de espessura da camada muscular. Enquanto os demais deram ênfase na FC e FR, foram citados também em alguns artigos o lactato, a pressão arterial e a creatina fosfoquinase, e todos com valores de alteração não significativos ou não apresentaram mudança.

Como desfecho para seguranças referentes aos artigos analisados, em todos os artigos não foram constatados efeitos adversos. Stefanou, et al. (2016) aponta que houve efeitos benéficos sistêmicos na microcirculação que não ocorreram distúrbios eletrocardiográficos e hemodinâmicos durante as sessões, que houve aumento da FC, FR, lactato e na creatina fosfoquinase (CPK), porém com valores não relevantes que alterassem a clínica do paciente e que a PAM não mudou, enquanto Sachetti, et al. (2018) e Akar, et al. (2015) não relataram diferença significativa entre os grupos que analisaram. No estudo de Fisher, et al. (2016) a aplicação da EENM não teve efeito significativo na espessura da camada muscular (ECM) porém havendo resultado positivo na recuperação da força muscular. Silva, et al. (2017) relataram, ao final de seus resultados, que não houve alterações significativas no lactato sérico e tampouco da creatina fosfoquinase (CPK) ao longo do estudo, no entanto apresentando mudança considerável nos valores de saturação venosa central de oxigênio $(\mathrm{SvcO} 2)$ no terceiro e último dia da aplicação da EENM.

Silva, et al. (2017) relataram que todos os pacientes foram sedados, porém 15\% deles manifestaram algia, havendo a interrupção da sessão, o que impossibilitou o acréscimo da intensidade, que foi padronizada e fixada a partir da maior contração muscular visível. Em nenhum artigo analisado foi relatado a ocorrência de queimaduras. Dessa forma, apresentando segurança da aplicabilidade da EENM para ser aplicada em pacientes críticos.

No Quadro 1 encontram-se as descrições gerais sobre cada artigo, listando números de pacientes envolvidos e estudados, as divisões dos grupos, parâmetros de intervenção, local de aplicação e desfecho de segurança encontrados. 
Research, Society and Development, v. 11, n. 1, e20711124754, 2022

(CC BY 4.0) | ISSN 2525-3409 | DOI: http://dx.doi.org/10.33448/rsd-v11i1.24754

Quadro 1: Descrição das informações contidas nos artigos selecionados.

\begin{tabular}{|c|c|c|c|c|c|c|}
\hline Estudo & Grupos & Pacientes (n) & Objetiro Principal do estudo & Parâmetros & Local de Aplicação & Desfecho para segurança \\
\hline STEFANOU et al, 2016 & $\begin{array}{l}\text { G1:Alta frequência } \\
\text { G2:Média } \\
\text { frequência }\end{array}$ & $\begin{array}{l}32 \text { pacientes com idade média } \\
\text { de } 58 \text { anos na UTI em VMe } \\
\text { sépticos, sedados ou não } \\
\text { sedados. }\end{array}$ & $\begin{array}{l}\text { Explorar os efeitos induzidos } \\
\text { pela NMES na mobilização de } \\
\text { cellulas progenitoras endoteliais } \\
\text { em pacientes sépticos em UTI. }\end{array}$ & $\begin{array}{l}\text { Impulso bifásico e largura de } \\
\text { pulso de } 400 \text { us; intensidade } \\
\text { máxima toletada; aplicada } \\
\text { uma única sessão com } \\
\text { duração de } 30 \text { minutos mais } \\
10 \text { minutos entre } \\
\text { aquecimento e recuperação. }\end{array}$ & $\begin{array}{l}\text { Nos pontos motores dos } \\
\text { músculos Vasto Lateral, } \\
\text { Vasto Medial e Fibular } \\
\text { Longo dos MMII, } \\
\text { bilateralmente. }\end{array}$ & $\begin{array}{l}\text { Efeitos benéficos sistêmicos na } \\
\text { microcirculaçào; Não ocorreram } \\
\text { distúrbios eletrocardiograficos e } \\
\text { hemodinâmicos durante as sessōes; } \\
\text { Em ambos os grupos houve aumento } \\
\text { da FC, FR, Lactato e na Creatina } \\
\text { Fosfoquinase mas com valores nẫo } \\
\text { significativos; Enquanto a PAM não } \\
\text { mudou. }\end{array}$ \\
\hline SILVA ef al, 2017 & $\begin{array}{l}\text { Todos os pacientes } \\
\text { fazendo uso da EENM }\end{array}$ & $\begin{array}{l}11 \text { pacientes criticos fazendo } \\
\text { uso de VM por } 24 \text { a } 48 \text { horas } \\
\text { com Politrauma (9), exceto com } \\
\text { fraturas em MMII, Pancreatite } \\
\text { (1) e cirurgia abdominal (1). }\end{array}$ & $\begin{array}{l}\text { Avaliar a segurança e viabilidade da } \\
\text { eletroestimulaçá neuromuscular. } \\
\text { Protocolo baseado na excitabilidade } \\
\text { neuromuscular e aplicado em } \\
\text { numerosos grupos musculares de } \\
\text { pacientes criticos. }\end{array}$ & $\begin{array}{l}\text { A EENM foi realizada uma vez ao } \\
\text { dia por } 15 \text { minutos ( } 90 \text { contraçóes) } \\
\text { por } 3 \text { dias consecutivos, frequência } \\
\text { de } 100 \mathrm{~Hz} \text {, tempo ON } 5 \mathrm{~s} \text {, tempo } \\
\text { OFF de } 5 \mathrm{~s} \text {, sem tempo de subida e } \\
\text { decaimento.A intensidade da largura } \\
\text { de pulsol foi padronizada e fixada a } \\
\text { partir da maior contraçăo muscular } \\
\text { visivel. }\end{array}$ & $\begin{array}{l}\text { Nos pontos motores dos } \\
\text { músculos glúteo máximo, } \\
\text {,quadriceps, tibial anterior, } \\
\text { isquiotibiais e } \\
\text { gastrocnêmios, } \\
\text { bilateralmente. }\end{array}$ & $\begin{array}{l}\text { No final de cada sessão de } 15 \text { min. de EENM, todos } \\
\text { os pacientes apresentaram fadiga com capacidade de } \\
\text { contraç̃o diminuida. Não houve alteraçóes } \\
\text { significativas no lactato serico e tampouco da } \\
\text { creatina fosfoquinase (CPK) ao longo do estudo.No } \\
\text { entanto, inicialmente nenhum paciente mostrou } \\
\text { alteraçấo dos valores de SvcO2 nos dois primeiros } \\
\text { dias, havendo mudança considerável no terceiro dia } \\
\text { da aplicaçào da EENM. Mostrando-se que o } \\
\text { protocolo aplicado e totalmente eficaz e seguro. }\end{array}$ \\
\hline SACHETTI ef al, 2018 & $\begin{array}{l}\text { G1(10): Grupo interveça: } \\
\text { EENM + Fisioterapia } \\
\text { convencional } \\
\text { G2(14): Gupo controle: } \\
\text { EENM Placebo + } \\
\text { fisioterapia convencional }\end{array}$ & $\begin{array}{l}24 \text { pacientes Fazendo uso VMI, } \\
\text { porém não fizeram aplicação de } \\
\text { bloqueadores neuromusculares } \\
\text { ou corticoides. }\end{array}$ & $\begin{array}{l}\text { Avaliar a segurança da técnica de } \\
\text { EENM em pacientes criticos em } \\
\text { VMI. O tratamento ocorreu desde a } \\
\text { entrada do paciente no estudo, } \\
\text { ainda sedado, iniciando } 24 \text { horas } \\
\text { após intubacâa até o momento da } \\
\text { extubaçẫo. }\end{array}$ & 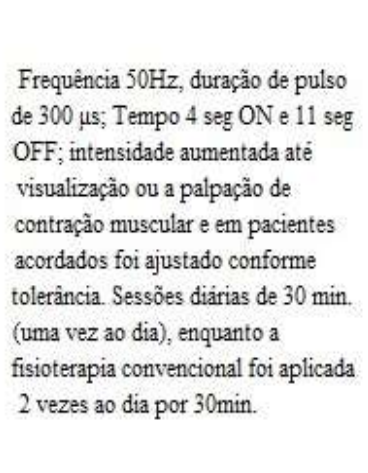 & $\begin{array}{l}\text { Nos pontos motores, } \\
\text { bilateralmente, dos musculos } \\
\text { Peitoral Maior e Reto } \\
\text { Abdominal. }\end{array}$ & $\begin{array}{l}\text { Foram avaliados Lactato sanguineo, } \\
\text { complacência pulmonar dinâmica e } \\
\text { variaiveis cardiorespiratórias (FC, FR, } \\
\text { saturação de oxigênio e Pressão arterial } \\
\text { antes e após a EENM). Não foram } \\
\text { observados eventos adversos; As } \\
\text { variäveis metabólicas não apresentou } \\
\text { diferença entre os grupos; Os niveis de } \\
\text { lactato sanguineo eda complacência } \\
\text { pulmonar dinàmica não houve diferença } \\
\text { significativa; Näo foi apresentado } \\
\text { alteraçōes nos paràmetros } \\
\text { cardiorespiratórios, quando avaliados } \\
\text { antes e após a EENM, apresentando-se } \\
\text { constantes. }\end{array}$ \\
\hline
\end{tabular}

(Continua) 
Research, Society and Development, v. 11, n. 1, e20711124754, 2022

(CC BY 4.0) | ISSN 2525-3409 | DOI: http://dx.doi.org/10.33448/rsd-v11i1.24754

\begin{tabular}{|c|c|c|c|c|c|c|}
\hline Estudo & Grupos & Pacientes (n) & Objetiro Principal do estudo & Parâmetros & Local de Aplicação & Desfecho para segurança \\
\hline AKAR et al 2015 & $\begin{array}{l}\text { G1: Exercicios ativos e EENM } \\
\text { G2: EENM } \\
\text { G3: Exercicios ativos }\end{array}$ & $\begin{array}{l}30 \text { pacientes conscientes entre } \\
\text { homens e mutheres em terapia } \\
\text { intensiva com DPOC. }\end{array}$ & $\begin{array}{l}\text { Investigar o impacto de ativos } \\
\text { mobilizaçao de extremidades e } \\
\text { estimulaçao elétrica neuromuscular } \\
\text { (EENM) desmame, alta hospitalar e } \\
\text { mediadores inflamatórios na DPOC } \\
\text { pacientes fecebendo ventiliaçà } \\
\text { mecanica. }\end{array}$ & $\begin{array}{l}\text { Frequencia } 50 \mathrm{~Hz} \text {; amplitude } 20 \mathrm{~mA} \text { e } \\
25 \mathrm{~mA} \text {; impulso bifásico, quadradoe } \\
\text { simétrico; duraçào de } 1,5 \text { ONe e } 0,75 \\
\text { OFF; aplicação a partir do } 2^{\circ} \text { dia de } \\
\text { VMI até a saida da UTI. }\end{array}$ & $\begin{array}{l}\text { Múscullos Delttöide e } \\
\text { Quadriceps bilateralmente. }\end{array}$ & 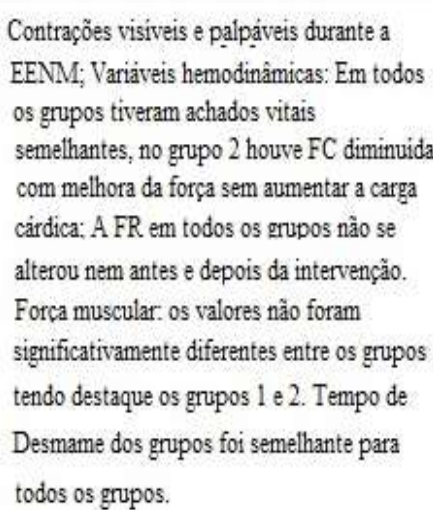 \\
\hline FISHER et al. 2016 & $\begin{array}{l}\text { Grupo intervenção fazendo } \\
\text { uso EENM } \\
\text { Grupo controle EENM } \\
\text { placebo }\end{array}$ & $\begin{array}{l}54 \text { pacientes criticos após } \\
\text { cirurgia cardiotorácica }\end{array}$ & $\begin{array}{l}\text { Investigar se a EENM impede a perda } \\
\text { de espessura da camada muscular } \\
\text { (ECM) e força e observar a variaçáo } \\
\text { temporal do ECMe força desde o dia } \\
\text { pré-operatório até o hospital descarga. }\end{array}$ & $\begin{array}{l}\text { Frequência de } 6 \mathrm{~Hz} \text {, estimulador } \\
\text { fornecido bifisico pulsos retangulares de } \\
0,4 \text { ms, os músculos foram estimulados } \\
\text { duas vezes ao dia }(2 \times 30 \text { minutos de } \\
\text { EENM com um intervalo de pelo menos } \\
30 \text { minutos entre as duas sessós, } 7 \text { dias } \\
\text { por semana durante toda a estadia na } \\
\text { UTI. }\end{array}$ & $\begin{array}{l}\text { A eletroestimulação foi } \\
\text { aplicada noo pontos } \\
\text { motores dos musculos } \\
\text { quadriceps bilateralmente } \\
\text { (os demais musculos foram } \\
\text { medidos pelo MRC), } \\
\text { desde o } 1^{\circ} \text { dia de pós } \\
\text { operatório até a alta da } \\
\text { UTI até no máximo } 14 \\
\text { dias. }\end{array}$ & $\begin{array}{l}\text { EENMn nào teve efeito significativo no } \\
\text { ECM, poém recuperarama forca } \\
\text { muscular } 4,5 \text { vezes mais rapido do que o } \\
\text { grupo controle. Na alta hospitalar todos } \\
\text { os pacientes recuperam niveis de forca do } \\
\text { pre-operatório, mas não de ECM. }\end{array}$ \\
\hline
\end{tabular}

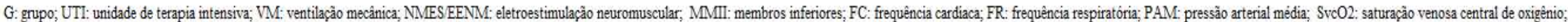
CPK: creatina fosfoquinase; VMI: ventiliaçoo mecâncica invasiva; DPOC: doença pulmonar obstrutiva crônica; $\mathrm{ECM}$ : espessura da camada muscular, $\mathrm{MRC}$ : medical research council.

Fonte: Autores (2021) 


\section{Discussão}

A presente revisão literária inclui apenas ensaios clínicos randomizados (ECR) que apresentaram as vantagens de fornecerem as causas da aplicabilidade da EENM em pacientes críticos com a variável segurança como efeito primário ou secundário do estudo. Além de revelarem-se com um desenho metodológico adequado para o desenvolvimento do presente estudo, esses artigos apontaram conclusões consistentes que sugeriram resultados positivos da EENM, não ocorrendo efeitos adversos que prejudicassem o quadro clínico dos pacientes e não apresentando impactos significativos na hemodinâmica quando aferidos e comparados antes e depois das sessões.

Mesmo que todos os artigos aqui incluídos e analisados tenham sido ECR, apenas o de Sachetti, et al. (2018) foi classificado como duplo cego, ou seja, com mascaramento dos voluntários e dos examinadores, e o de Fisher, et al. (2016) sendo classificado como único cego no qual os pacientes não foram informados quanto ao tratamento a ele designado.

Todos os estudos foram realizados com uma preocupação em comum pelo uso da EENM que seria o impacto sobre a hemodinâmica dos pacientes, porém nenhum estudo apresentou alterações clínicas relevantes, embora sendo demonstrado alguns aumentos marginais na aferição após sua administração como a FC, FR e do lactato mas não representando alterações que representassem uma anormalidade.

Segundo Cerqueira, et al. (2019), em seu estudo que caracterizou-se como ECR, foi exposto que não houve nenhuma alteração clínica nas variáveis hemodinâmica sobre a FC, PAS (pressão arterial sistólica), PAD (pressão arterial diastólica) e na PAM (pressão arterial média), como também não demonstrou arritmias cardíacas nos pacientes ao realizar a EENM e que nenhuma alteração foi encontrada nas variáveis respiratórias como FR e na SpO2 (saturação periférica de oxigênio), permanecendo dentro dos limites considerados normais.

Ainda de acordo com Cerqueira, et al. (2019), foi proposto que a EENM poderia ser usado de forma segura no póscirúrgico cardíaco como forma de amenizar o declínio da força e que, em estudos anteriores havia um anseio com relação ao uso da EENM nesses pacientes bem como os considerados críticos na área médica pela possível geração de arritmias.

Hill, et al. (2018), em sua revisão, relataram que, em relação aos eventos adversos, não há diferença de risco quanto à mortalidade dos pacientes quando aplicado a EENM isoladamente ou apenas tratamento físico convencional ou em conjunto, declarando que pacientes incapazes ou indispostos podem utilizar a eletroestimulação como uma estratégia de reabilitação, mas deixando claro que a qualidade de evidência é baixa.

Embora os estudos estabeleçam critérios para examinar se a hemodinâmica ou se a incidência de complicações estaria dentro da faixa de normalidade para demonstrar a segurança e a viabilidade do tratamento pela EENM, os parâmetros de aplicação, os seus protocolos e as características dos participantes foram altamente variáveis entre os estudos, dessa forma inviabilizando agrupar os seus resultados para que pudessem ser descritos.

Fazendo um comparativo, Chen, et al. (2016), em sua metanálise, evidenciaram a heterogeneidade dos programas de aplicação da EENM, no que tange a intensidade do estímulo. No entanto, concluíram que a mais adequada dosagem é a individualizada e a máxima tolerada. A respeito da sua aplicabilidade em pacientes com DPOC moderada à grave, constataram o aumento da força do quadríceps e da resistência ao exercício, porém não afirmaram melhora significativa na qualidade de vida.

A EENM foi implementada nos estudos e, como conclusão, a segurança da sua aplicação foi citada, demonstrando que sua aplicabilidade era bem tolerada pelos pacientes sem causar eventos adversos, sugerindo que é segura e viável mesmo que em pacientes críticos que requerem cuidados intensivos que precisam manter o status hemodinâmico aceitável.

Comparando com os resultados de Jones, et al. (2016), em sua revisão sistemática sobre a ocorrência de eventos adversos eles relatam que não houve nenhum que fosse considerado grave, porém alguns de seus estudos foram constatados que 1 a cada 
Research, Society and Development, v. 11, n. 1, e20711124754, 2022

(CC BY 4.0) | ISSN 2525-3409 | DOI: http://dx.doi.org/10.33448/rsd-v11i1.24754

20 pacientes apresenta desconforto muscular após as primeiras sessões e alguns poucos apresentaram surgimento de eritema persistente pelo uso de eletrodos adesivos, mas que os demais estudos não apresentaram eventos adversos, sugerindo que a EENM pode ser um tratamento bem tolerado, eficaz e seguro para fraqueza muscular.

Neste estudo, houve limitações quanto ao número de ensaios clínicos analisados e tamanho amostral reduzidos devido à falta de mais artigos que se detivesse na segurança da aplicabilidade da EENM em pacientes críticos nas unidades de terapia intensiva.

\section{Conclusão}

Os resultados das análises sugerem que a EENM pode ser implementada nas UTI's na recuperação e prevenção da polineuromiopatia, pois a ausência de efeitos secundários maléficos ou significativos enfatiza tal afirmação.

Entretanto estudos futuros devem explorar ainda mais o papel da EENM como um recurso alternativo e seguro para que a sua aplicabilidade clínica seja vista como importante nas UTI's e demonstre total segurança da aplicação da mesma sendo necessária a realização de estudos que utilizem protocolos bem estabelecidos com maior rigor estatísticos, com maiores amostras, com parâmetros mais uniformizados e que sua variação seja de maneira controlada para determinar a intervenção mais adequada afim de aumentar a qualidade metodológica dos ECR. Dessa forma, fortalecendo o que compreende como a "tríade da prática da fisioterapia”: melhor evidência disponível, experiência profissional (expertise) e a preferência do paciente.

Para tanto, é necessário observar os níveis de evidências. Por exemplo, a escala PEDro desenvolvida pela Physiotherapy Evidence Database e graus de recomendação da aplicabilidade da EENM com base na segurança e efetividade direcionando ao processo de reintegração social, laboral e familiar do indivíduo. Sendo de fundamental importância as constantes atualizações sobre as informações crescentes e criteriosas, pois elas servem de elementos norteador para a tomada de decisão clínica diminuindo as incertezas. Por fim, foi observado nas bases de literaturas pesquisadas, diversos trabalhos que utilizaram a EENM. Contudo, faz-se necessário novos estudos randomizados para melhor embasar a aplicabilidade desta técnica nas UTI’s.

\section{Referências}

Akar, O., Günay, E., Sarinc Ulasli, S., Ulasli, A. M., Kacar, E., Sariaydin, M., Solak, Ö., Celik, S., \& Ünlü, M. (2015). Efficacy of neuromuscular electrical stimulation in patients with COPD followed in intensive care unit. The Clinical Respiratory Journal, 11(6), 743-750. https://doi.org/10.1111/crj.12411

Cerqueira, T. C. F., Cerqueira Neto, M. L. d., Carvalho, A. J. G., Oliveira, G. U., Araújo Filho, A. A. d., Carvalho, V. O., Cacau, L. d. A. P., Silva Júnior, W. M. d., Mendonça, J. T. d., \& Santana Filho, V. J. d. (2019). Neuromuscular Electrical Stimulation on Hemodynamic and Respiratory Response in Patients Submitted to Cardiac Surgery: Pilot Randomized Clinical Trial. International Journal of Cardiovascular Sciences. https://doi.org/10.5935/2359-4802.20190028

Chen, R.-c., Li, X.-y., Guan, L.-1., Guo, B.-P., Wu, W.-1., Zhou, Z.-q., Huo, Y.-t., Chen, X., \& Zhou, L.-q. (2016). Effectiveness of neuromuscular electrical stimulation for the rehabilitation of moderate-to-severe COPD: a meta-analysis. International Journal of Chronic Obstructive Pulmonary Disease, Volume 11, 2965-2975. https://doi.org/10.2147/copd.s120555

Ferreira, L. L., Vanderlei, L. C. M., \& Valenti, V. E. (2014). [ARTIGO RETRATADO] Estimulação elétrica neuromuscular em pacientes graves em unidade de terapia intensiva: revisão sistemática. Einstein, 12(3), 361-365. https://doi.org/10.1590/s1679-45082014rw2955

Fischer, A., Spiegl, M., Altmann, K., Winkler, A., Salamon, A., Themessl-Huber, M., Mouhieddine, M., Strasser, E. M., Schiferer, A., Paternostro-Sluga, T., \& Hiesmayr, M. (2016). Muscle mass, strength and functional outcomes in critically ill patients after cardiothoracic surgery: does neuromuscular electrical stimulation help? The Catastim 2 randomized controlled trial. Critical Care, 20(1). https://doi.org/10.1186/s13054-016-1199-3

França, E. É. T. d., Ferrari, F., Fernandes, P., Cavalcanti, R., Duarte, A., Martinez, B. P., Aquim, E. E., \& Damasceno, M. C. P. (2012). Fisioterapia em pacientes críticos adultos: recomendações do Departamento de Fisioterapia da Associação de Medicina Intensiva Brasileira. Revista Brasileira de Terapia Intensiva, 24(1), 6-22. https://doi.org/10.1590/s0103-507x2012000100003

Godoy, M., Costa, H., Neto, A., Serejo, A., Souza, L., Kalil, M., Rodrigo, M., Monteiro, F., Souza, O., Freitas, M., Bastos, V., Novellino, P., Matta, A., \& Orsin, M. (2015). Fraqueza muscular adquirida na UTI (ICU-AW): efeitos sistêmicos da eletroestimulação neuromuscular. Rev. Bras. de Neurologia, 51(1). https://revistas.ufrj.br/index.php/rbn/article/view/3109

Hashem, M. D., Parker, A. M., \& Needham, D. M. (2016). Early Mobilization and Rehabilitation of Patients Who Are Critically Ill. Chest, 150(3), 722-731. https://doi.org/10.1016/j.chest.2016.03.003 
Research, Society and Development, v. 11, n. 1, e20711124754, 2022

(CC BY 4.0) | ISSN 2525-3409 | DOI: http://dx.doi.org/10.33448/rsd-v11i1.24754

Hill, K., Cavalheri, V., Mathur, S., Roig, M., Janaudis-Ferreira, T., Robles, P., Dolmage, T. E., \& Goldstein, R. (2018). Neuromuscular electrostimulation for adults with chronic obstructive pulmonary disease. Cochrane Database of Systematic Reviews. https://doi.org/10.1002/14651858.cd010821.pub2

Jones, S., Man, W. D.-C., Gao, W., Higginson, I. J., Wilcock, A., \& Maddocks, M. (2016). Neuromuscular electrical stimulation for muscle weakness in adults with advanced disease. Cochrane Database of Systematic Reviews. https://doi.org/10.1002/14651858.cd009419.pub3

Meesen, R. L. J., Dendale, P., Cuypers, K., Berger, J., Hermans, A., Thijs, H., \& Levin, O. (2010). Neuromuscular Electrical Stimulation As a Possible Means to Prevent Muscle Tissue Wasting in Artificially Ventilated and Sedated Patients in the Intensive Care Unit: A Pilot Study. Neuromodulation: Technology at the Neural Interface, 13(4), 315-321. https://doi.org/10.1111/j.1525-1403.2010.00294.x

Menezes, T. C. d., Bassi, D., Cavalcanti, R. C., Barros, J. E. S. L., Granja, K. S. B., Calles, A. C. d. N., \& Exel, A. L. (2018). Comparisons and correlations of pain intensity and respiratory and peripheral muscle strength in the pre- and postoperative periods of cardiac surgery. Revista Brasileira de Terapia Intensiva, 30(4). https://doi.org/10.5935/0103-507x.20180069

Moraes, A. V., Costa, J. S., \& Nascimento, J. M. R. (2019). The effects of transcutaneous electrostimulation in patients in the intensive care unit. Rev. Pesqui. Fisioter, 9(4) 572-580. https://pesquisa.bvsalud.org/portal/resource/pt/biblio-1151936

Pinheiro, A. R., \& Christofoletti, G. (2012). Fisioterapia motora em pacientes internados na unidade de terapia intensiva: uma revisão sistemática. Revista Brasileira de Terapia Intensiva, 24(2), 188-196. https://doi.org/10.1590/s0103-507x2012000200016

Puthucheary, Z., Montgomery, H., Moxham, J., Harridge, S., \& Hart, N. (2010). Structure to function: muscle failure in critically ill patients. The Journal of Physiology, 588(23), 4641-4648. https://doi.org/10.1113/jphysiol.2010.197632

Sachetti, A., Carpes, M. F., Dias, A. S., \& Sbruzzi, G. (2018). Safety of neuromuscular electrical stimulation among critically ill patients: systematic review. Revista Brasileira de Terapia Intensiva, 30(2). https://doi.org/10.5935/0103-507x.20180036

Sant'Ana, C. d. F., \& Castro, D. L. d. (2019). Ensino de Química em repositórios digitais: Uma análise de simuladores sob o viés da experimentação por investigação. Research, Society and Development, 8(2), Artigo e1382588. https://doi.org/10.33448/rsd-v8i2.588

Santos, L. S., Ribeiro, E. S., Neves, V. A., Silva, M. H. M. d. L., Silva, C. F., \& Gama, G. L. (2020). Uso de agentes eletrofísicos por fisioterapeutas no Brasil. Research, Society and Development, 9(12), Artigo e30591210965. https://doi.org/10.33448/rsd-v9i12.10965

Santos, L. J. d., Silveira, F. d. S., Müller, F. F., Araújo, H. D., Comerlato, J. B., Silva, M. C. d., \& Silva, P. B. d. (2017). Avaliação funcional de pacientes internados na Unidade de Terapia Intensiva adulto do Hospital Universitário de Canoas. Fisioterapia e Pesquisa, 24(4), 437-443. https://doi.org/10.1590/18092950/17720924042017

Santos, R., Carvalhais, V., Paz, C., \& Criollo, C. (2015). Use of functional electrical stimulation post-stroke: systematic review. Revista Neurociências, 23(01), 103-115. https://doi.org/10.4181/rnc.2015.23.01.1008.13p

Silva, P. E., Babault, N., Mazullo, J. B., de Oliveira, T. P., Lemos, B. L., Carvalho, V. O., \& Durigan, J. L. Q. (2017). Safety and feasibility of a neuromuscular electrical stimulation chronaxie-based protocol in critical ill patients: A prospective observational study. Journal of Critical Care, 37, 141-148. https://doi.org/10.1016/j.jcrc.2016.09.012

Stefanou, C., Karatzanos, E., Mitsiou, G., Psarra, K., Angelopoulos, E., Dimopoulos, S., Gerovasili, V., Boviatsis, E., Routsi, C., \& Nanas, S. (2016). Neuromuscular electrical stimulation acutely mobilizes endothelial progenitor cells in critically ill patients with sepsis. Annals of Intensive Care, 6(1). https://doi.org/10.1186/s13613-016-0123-y 